\title{
Statistical Approach in Power Cables Diagnostic Data Analysis
}

\author{
Piotr Cichecki, Rogier A. Jongen, Edward Gulski, Johan J. Smit \\ Delft University of Technology, High Voltage Technology and Management, Mekelweg 4, 2628CD Delft, The Netherlands
}

\author{
Ben Quak \\ Seitz Instruments AG, Mellingerstrasse 12, 5443 Niederrohrdorf, Switzerland \\ Frank Petzold \\ SebaKMT, Dr. Herbert Iann Str.6, 96148 Baunach, Germany \\ and Frank de Vries \\ Nuon Tecno, Voltastraat 2, 1800 AJ Alkmaar, The Netherlands
}

\begin{abstract}
This paper describes the application of statistical analysis to the on-site diagnostic data of HV power cables to set-up knowledge rules and measuring criteria for particular cable insulations and accessory (joints, terminations). Such approaches can successfully be employed later in Asset Management decision processes. In this paper based on different cable data populations of two diagnostic parameters like: partial discharges and $\tan \delta$, statistical analysis has been applied. Besides this, several practical examples also have been presented
\end{abstract}

Index Terms - Cable insulation, data analysis, partial discharges, statistical analysis.

\section{INTRODUCTION}

IN recent years growing interest in non-destructive and advanced diagnostic systems resulted in several modern diagnostic methods with output data suitable for statistical analysis. Analysis of diagnostic data of medium voltage (MV) and high voltage (HV) power cables especially from on-site measurements is a complex process. This is even more difficult when diagnostic input data can not be verified by the general knowledge of particular insulation types or e.g. component types. It is known that the analysis of PD processes and dielectric losses can reflect the aging condition of cable insulation and also by the evaluation of maximum values, e.g. PD amplitude or dielectric losses can be used to estimate borders between acceptance limits. Therefore diagnostic parameters like: partial discharge (PD) amplitude, PD inception voltage (PDIV) or dielectric losses represented by $\tan \delta$ or $\Delta \tan \delta$ can be used for statistical processing [1-5]. As a result, obtaining experience norms for diagnostic data (PD or dielectric losses) including confidence boundaries will help to evaluate the insulation condition of new or service power cable systems. Having such boundary values can support asset management (AM) decision processes e.g. about the type and intervals of maintenance inspections or replacement schedule [3]. Moreover, if failure data is available, statistical failure analysis can be a powerful tool to

Manuscript received on 14 April 2008, in final form 6 September 2008. determine whether replacement is necessary now or in the future, to obtain a certain reliability of the network [4-5].

Beside this, diagnostic data obtained on-site plays an important role because it:

- gives information about actual cable condition (on-site measurements - result directly or after measurements),

- gives the possibility to estimate norm values for particular cable components,

- supports the maintenance and operation decision processes for maintenance/replacement of power cable systems,

- helps to develop knowledge rules in cable data interpretation as well as cable insulation aging processes, With diagnostic information as obtained using on-site diagnostic methods, all combined results properly interpreted can be used for a classification of the cable systems.

One of the major goals in on-site testing of service aged MV/HV power cables in the coming years will result with more attention put to decisions which have to be taken about maintenance or replacement of the oldest serviced cables circuits. Those circuits are reaching their life limits and their reliability must be predicted based on actual diagnostics results [2]. In general, such strategic decisions are the responsibility of the asset management [6]. According to [5] the following chart of analysis procedures is presented in Figure 1. Based on information about the present and future asset performance, characteristics e.g. technical condition and the knowledge about degradation status inject additional 


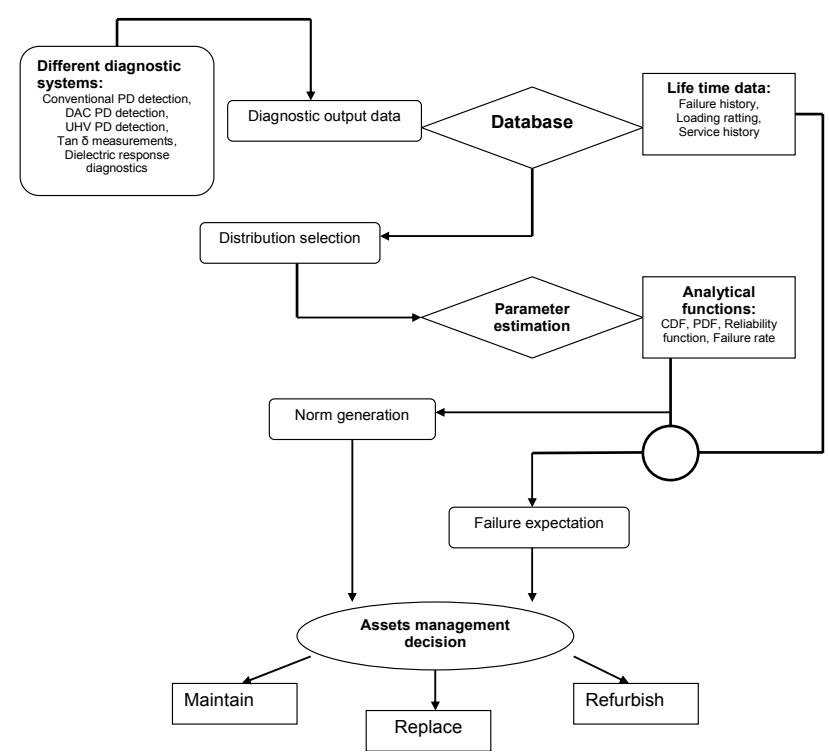

Figure 1. Asset management decision process.

criteria when decisions about maintenance or replacement have to be taken. However, at present such AM strategies may meet several difficulties due to: very low numbers of failures, no cable service history availability. In the case where no statistical predictions are possible or prediction has to be limited because of low quality data (data not complete) [3].

With regards to degradation processes HV cables are more complex than MV power cables and the systematic knowledge about the actual aging needs further investigations and field verifications. Another problem is the lack of HV power cables fixed diagnostic rules for on-site condition diagnostics. Because of these reasons for few last years in the field of on-site cable diagnostic, efforts are put to systematize measuring procedures and test criteria [7]. This mostly regards to clarification the rules and guidelines for applied voltage ratings and limitations for old and new circuits. Performing on-site diagnostics on similar types of components typical "limits" like PD amplitude, at Uo, or 1.7 Uo, or the maximum acceptable dielectric losses range can be set for a particular component e.g. insulation type in certain age or condition. This approach can help develop reference database which can be used to reflect typical practical condition situations:

Status A: New or aged: no defects or aging symptoms which means the minimal maintenance for optimal availability.

Status A/B: Aged: degradation observed, possibly harmful defects present which means: no impact on reliability, keep in status B, against minimal cost and maintain optimal availability. But it also could be that the reliability is decreased which means: refurbish to status B, or if this is not possible, stabilise to prevent quick aging, maintain optimal availability, by periodical condition assessment.

Status C/D: Significantly aged: very degraded, serious defects present which means very low reliability, instable situation, end as quick as possible. But it also could be that no operation is recommended which means: maintenance aimed to prevent environmental pollution and safe conservation, extension of life time or availability is no issue.
Developed in this way knowledge rules and measuring experience provided with large numbers of data will help to setup service criteria for different cable types as well as cable accessories.

In this paper special efforts were put to highlight the statistical approaches in cable diagnostic analysis and to show briefly the processes of distribution data fitting, distribution selection based on data type and norm value generation for PD parameters and $\tan \delta$ parameter. Further on in this paper several examples of estimated norm values based on on-site measurements will be presented.

\section{ON-SITE INSULATION AND ACCESSORIES CONDITION ASSESSMENT}

The insulation failures in a cable network may be caused by:

- lower dielectric strength due to aging processes,

- by internal defects in the insulation system,

- an external factors like e.g. poor workmanship installation or assembling works (missing insulation screen at the cable/joint transition or cable splice improperly prepared (cable splice not round) especially XLPE cables.

It is known that on-site voltage testing in combination with measurements of the dielectric properties may give an indication about the actual condition of service aged cable insulation. For MV/HV power cables different on-site inspections/diagnostics are available. Thus, different diagnostic approaches to output data regarding to short and long-term condition estimations exists $[1,2,4-6]$. The results of non-destructive, on-site measurements may have a direct relation to the average qualitative level of the insulation at the moment of measurement and can thus be applied as a trend or fingerprint measurement during future inspections.

Based on the last 20 years of on-site experience in power cable testing it follows from [4-7] that PD diagnosis and integral dielectric diagnostics represented by $\tan \delta$ may play an important role in short and long-term condition assessment. In the majority of examples the PD diagnosis may indicate weak spots in a cable connection and cable insulation itself. With XLPE installation, usually if PDs are recognized they are coming from joint or termination related problems as a result of poor workmanship, unlikely to originate in the insulation itself. The occurrence of partial discharges have physical character and it is described by such important parameters as PD inception voltage, PD pulse magnitudes, PD patterns and PD site location in a power cable. Performing onsite PD measurements at test voltages higher than Uo (normal network voltage) stresses up to 2 Uo is important to (Figure 2):

a) inspect if there are discharging insulation defects with $\mathrm{PD}$ inception voltage (PDIV) $>$ Uo. Such defects may initiate insulation failure in the case of single phase earth fault (non-earthed system) at voltages e.g. 1.7 Uo,

b) confirm in the same way as in a successful after-laying test, that there are no PD detected up to 2 Uo and that 
during the service operation the power cable insulation is free of discharging defects,

c) the on-site test has not initiated any discharging processes in the insulation which information is important to confirm the non-destructives of the diagnostic on-site test itself. PD diagnostics based on analysis of measured PD parameter like (PD magnitude, PD intensity and PDIV) results in an

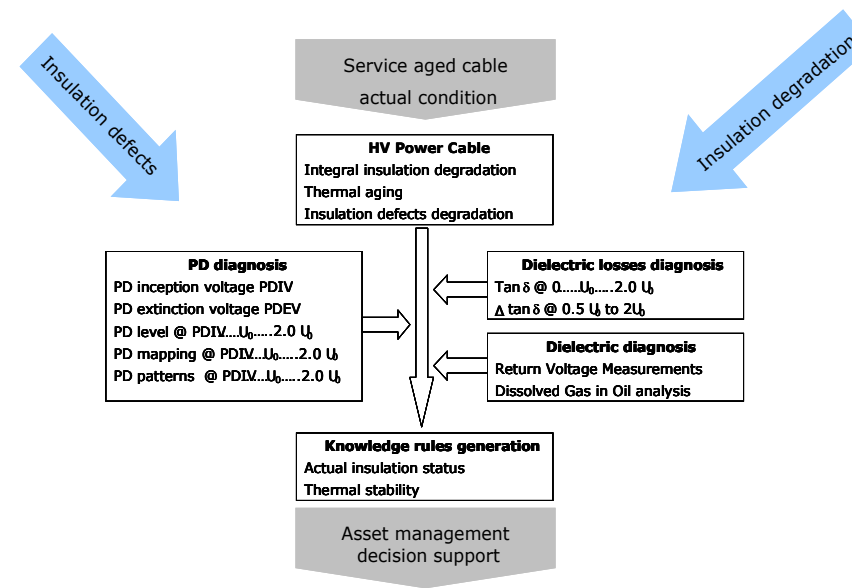

Figure 2. Important diagnostic parameters and knowledge rules generation goals.

overall cable condition assessment model which can be developed.

In this case the model of condition assessment is mainly based on three correlated PD parameters mentioned above. It is common known that the PD inception voltage (PDIV) is a good indicator to monitor the degradation of the insulation of high voltage components. Experience has shown that the PDIV might decrease due to aging phenomena [3,4,5-8].

This symptom depends on several factors like e.g. type of insulation, defect, and defect volume. Also decrease of PDIV $<$ Uo is directly related to an increase of PD intensity. Thus, the monitoring of the PDIV over an extensive period of the high voltage component and correlated PDs is important in aging phenomenon controlling. Another parameter is discharge magnitude which can be related to the defect size and the defect volume that is occupied by a discharging. The partial discharge magnitude is thus a good indication for both the energy and volume of a discharge source. On the other hand the attenuation of PD pulse magnitude depending on cable the length and insulation thickness which always should be considered. Due to aging processes (e.g. a repeated partial discharge bombardment) the volume of a discharge is expected to grow (e.g. treeing, growing cavities, etc). Therefore increasing partial discharge magnitudes is also an indicator of aging. The partial discharge intensity is called the partial discharge occurrence frequency, within a component at a particular spot in the insulation. Degradation of the insulation in high voltage equipment can be reflected by the energy content of the partial discharge bombardment. The total amount of energy dissipated is directly related to the frequency and the magnitude of the partial discharges.

Partial discharge sources need a starting electron to ignite, in aged sources a greater number of electrons may be available due to protrusions, impurities in the insulation and micro particles caused by aging (e.g. electrical or mechanical stress).
Therefore the partial discharge intensity can also be an indicator for aging condition assessment

Unless the fact that all power cables have high voltage life expectancy, during the whole service life $>30$ years they are subjected to thermal, mechanical and environmental stresses. All these influences raise the working temperature of the power cable. As a result higher temperature accelerates chemical reactions resulting in degradation of dielectric characteristics by e.g. polarization processes in the paper or oxidation processes in oil or in extreme case braking cellulose bonds. Such increase of degradation results in increases of loss factor and further degradation. Therefore measurement of dielectric losses at operational power frequencies is important issue to avoid failures at relatively high operating field strength of HV power cables.

The dielectric losses measurement ( $\tan \delta$ ) can be applied in the determination of the loss factor of the insulation material [7-9]. Due to the fact that this factor increases during the ageing process of the cable, the tan $\delta$. measurement should be regarded as a diagnostic and/or supporting measurement. In practice, for $\mathrm{HV}$ insulation it is known that in addition to absolute value of $\tan \delta$ at certain test voltage also the increment of tan delta as measured at two designated voltages so called $\Delta \tan \delta$ or tip-up is important for condition assessment. The loss tangent can be measured as a function of voltage to check the quality of impregnation. The $\tan \delta$ value of a cable is strongly influenced by the composition of the connection, the trace, and the deviations in joints and the actual measurement is only applicable as a trend measurement if composition circumstances of the trace and thermal conditions of successive measurements are virtually identical. For HV paper insulated cables the $\tan \delta$. can be an important indicator of possible thermal breakdowns. In particular for paper-oil insulated power cables this is of importance to determine also the degradation ratio of the paper. Relaying on the dielectric properties, mainly polarization of the insulation, the dielectric response on interaction between macroscopic polarization and the electric field is good a representation of the time dependency of the polarization processes in the insulation.

\section{STATISTICAL ANALYSIS - DIAGNOSTIC DATA PROCESSING}

It is known from the praxis that the data available from the diagnostic inspections of $\mathrm{MV} / \mathrm{HV}$ components can be characterized as follows:

1. for one particular type of the component, small populations of data can be available,

2. the data available for one particular type can be influenced by factors like: service life, time moment of inspection and maintenance history.

These two aspects have to be taken into account in different ways. The first method, applicable for representative population sizes involves the process of distribution fitting and norm value generation based on distribution. The second method applicable for small populations of data involves norm 
generation bases upon so called non-parametric calculating [10]. The statistical analysis than can be divided in two different ways. The second approach is the analysis based on non-parametric methods. With this method the data can be analysed based on histograms of the failure data. The empirical cumulative distribution function (CDF) is based on the histogram and has no underlying mathematical distribution. A disadvantage is that predictions which lay outside the range of observations are not possible. Although this method can give valuable information it is not further discussed in this paper. Another approach is based on parametric methods. With this method statistical distributions are used to fit the data and to estimate the accompanying parameters. The distributions or probability models are used to explain the behaviour of the breakdown processes or the life time data. The processes can be described as random events which result in random variables which can have a discrete or continuous character $[10,11,12]$. Life data, reliability parameters and in fact the results of the majority of measurements, are examples of continuous variables. Examples of discrete random variables are the number of faults/breakdowns following the application of a voltage of given discrete shape and duration. For the statistical evaluation of data obtained on HV components, the most popular models/distributions are:

- In the case of discrete random variables: binomial- or Poisson distributions,

- In the case of continuous random variables: e.g. Normal, Log-Normal, Exponential, Weibull and Gumbel distributions.

Diagnostic data like PD amplitude as a function of test voltage and life time data can be regarded as continuous data, for this reason, the discrete models are not further taken into account.

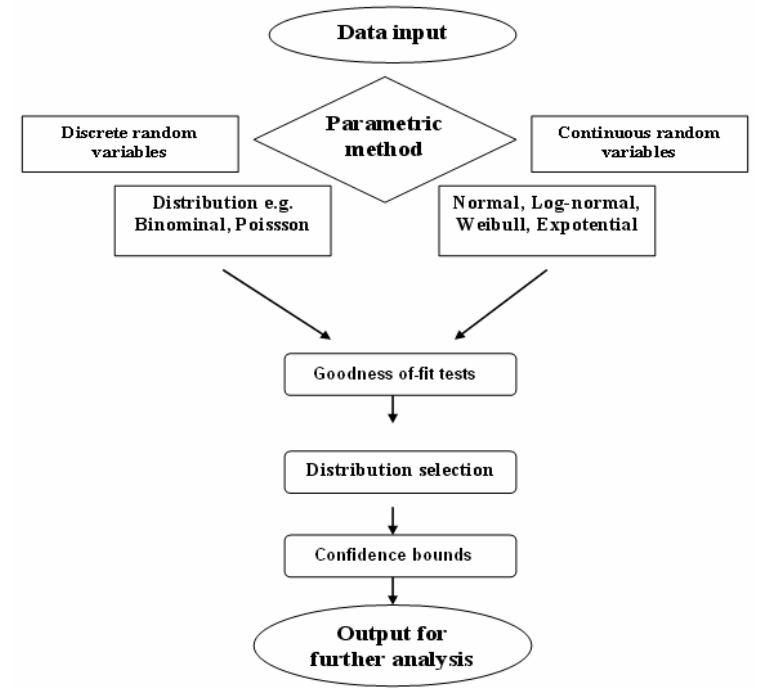

Figure 3. Basic steps of performing the statistical analysis on data.

The statistical analysis is performed as shown in the flowchart of Figure 3.

In the figure it can be seen that several distributions can be used to fit the continuous random data. With goodness-of-fit tests it can be seen which distribution fits the data best. When the appropriate distribution is chosen the confidence bounds of the distribution can be obtained. These bounds show the variation of a fitted distribution with a certain required reliability. In general: if more input data (more samples) are available this results in smaller confidence bounds (with respect to the same level of reliability).

\subsection{DISTRIBUTION FITTING}

Distribution fitting means that the data points from the diagnostic data are best fitted with an appropriate distribution. The horizontal axis represents the failure time, age or

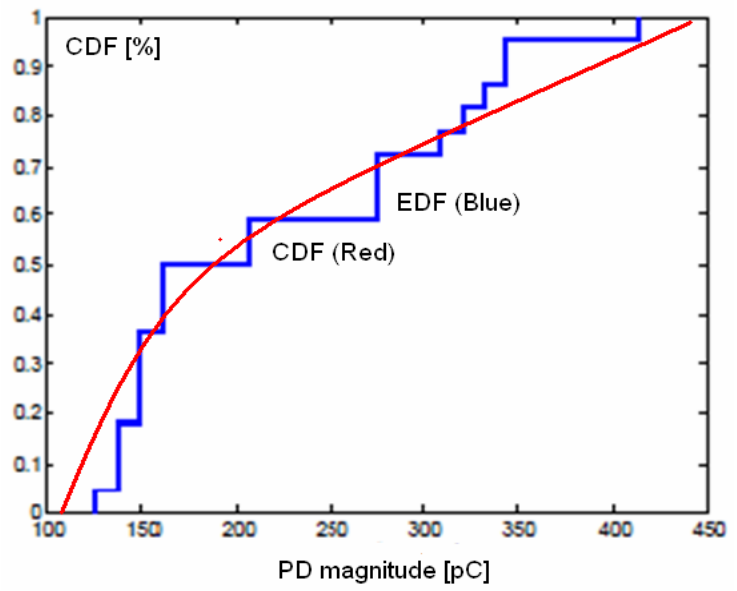

Figure 4. Empirical density function (EDF).

diagnostic parameter of the components and the vertical axis represents the unreliability $\mathrm{F}(\mathrm{t})$ in percentages (Figure 4).

Parametric distribution fitting has to be done in a number of steps where matching a dataset to a particular statistical failure distribution is done. After that to characterize the particular distribution, using selected statistical parameters the boundaries e.g. $1-\sigma, 2-\sigma$ or $3-\sigma$ can be calculated. In probability and stochastic process theory, a statistical distribution can be described by a number of different formulas [12-13]. Each formula is related to the other. The probability density function (PDF) and the cumulative density function (CDF) are the most basic formulas and will be used to denote the failure distributions. Usually these formulas are denoted by:

$F(t)$, for the $C D F$

$f(t)=d F / d t$ for the PDF

Another related term is the hazard rate, which describes the conditional probability that system or component fails within the interval $(\mathrm{t}, \mathrm{t}+\Delta \mathrm{t}]$. The hazard is related to the CDF and PDF via:

$z(t)=f(t) / R(t)$, with $R(t)=1-F(t)$

The function $R(t)$ is also known as the reliability function. Normally the formulas describing a statistical distribution are functions of time, $t$. In the analysis of the diagnostic data generated by the measuring systems time is replaced by partial discharge or dielectric losses parameter $(\tan \delta)$. 


\subsection{PARAMETER ESTIMATION}

When a distribution is chosen to fit the data, the accompanying parameters have to be estimated. Different parameter estimation methods can be used for this. One of them is a part of the distribution fitting as described above. The parameters can be obtained from the plot of the fitted CDF. More sophisticated methods are the least square estimation and the maximum likelihood estimation (MLE). Both methods can give different results. Based on the data one method can be preferred over another one.

\subsection{GOODNESS-OF-FIT TESTS}

After the parameter estimation of the distribution is done, it has to be considered how well it fits the data points. This can be done by several goodness-of-fit tests. Basically the procedure involves calculating the cumulative density functions of the distributions to be tested and then comparing these CDF's with the empirical density function (EDF) of the dataset. A picture of an EDF is shown in Figure 4. For illustrative purposes a rather small dataset is used.

The comparison between the CDF and EDF can be done based on different goodness - of - fit tests. When the least square estimation is used the correlation coefficient can be obtained. It describes the distance between the data points and the fitted distribution. Other tests which can be used for both estimation methods are e.g. the Kolmogorov-Smirnov test, the Chi-squared test and Anderson-Darling test. The results of the tests can be used to compare the fit of different distributions with each other and finally the best fitting distribution can be selected.

It is important to note that visual verification while choosing the best fitting distribution is very useful. In some instances there is so little difference between the distributions based on the calculated statistics that visual inspection is necessary to make the final decision.

Figure 5 and Figure 6 show an example of the result of the whole fitting procedure. Figure 5 shows the EDF of the measured data (blue line), also the estimated best fitting distribution, in this case a lognormal distribution, is depicted. Figure 6 shows the measured data (green bars) compared to the fitted distribution (blue line). In this figure

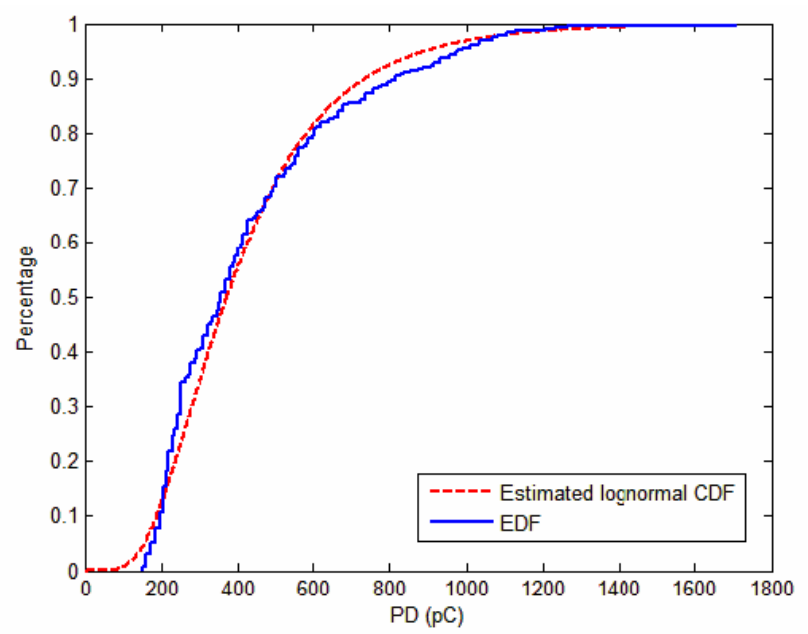

Figure 5. Comparison between the estimated CDF of a lognormal distribution and the EDF of measured data (example).

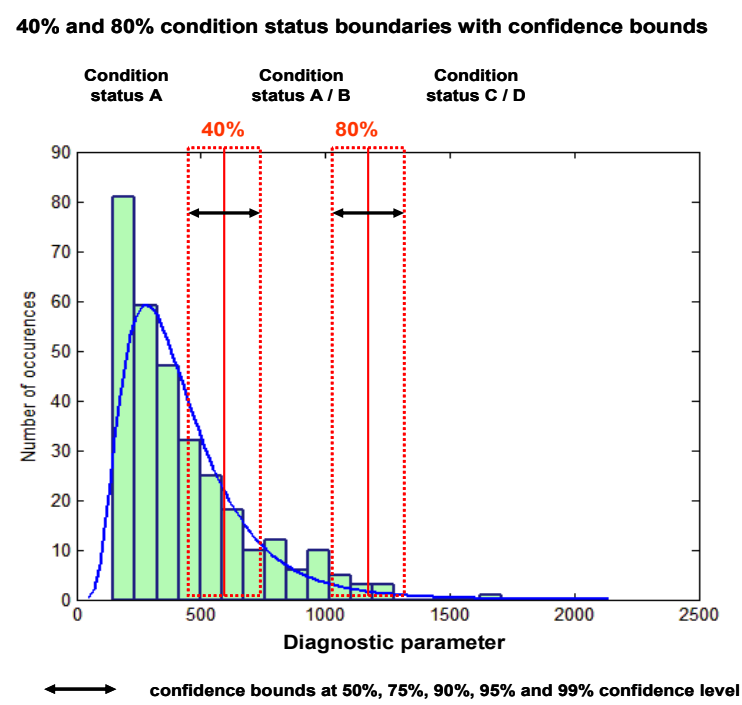

Figure 6. Example of PDF of the estimated lognormal distribution compared to the histogram of obtained data. Red lines represent boundary settings for particular diagnostic parameter.

the fitted distribution can be compared with the histogram of the data.

\subsection{BOUNDARY ESTIMATION (DECISION SUPPORT)}

The interpretation of diagnostic data can be difficult when the boundaries are not well known. For example PD measurement on a paper insulated cable gives a certain magnitude for a discharging defect and it does not necessarily has to result in a concern about the quality of the cable. To get more insight in the results of the measurements a statistical analysis on the PD values of different PD measurements on the same type of cable has to be performed. In this way a distribution can be obtained which describes the measured values obtained till so far. This can be used to classify the cable if certain boundary values are selected. This can be PD level at e.g. $40 \%, 80 \%$ or $95 \%$ of the distribution. When this boundary value or more is measured an advice can be given to test the cable again in a short time (to monitor if an increase can be seen) or to replace the cable section with a new cable.

Before last decision it has to be investigated if the population or a part of it can fulfil the reliability as requested by the asset manager. By means of the fitted cumulative distribution function the age of a component together with the reliability can be obtained. This is done by evaluating the B-lives. These indicate a certain level of reliability based on the age of a component. In the world of engineering the used B-life starts mostly around B10. This means that $10 \%$ of the total population will fail at a certain age (or with certain parameter value) and that $90 \%$ survives. Different values can be used as an acceptance value for unreliability/reliability depending on the criticality of a failure, cable component. When the probability of failure gets too high, components with the PD amplitude or dielectric losses higher then the chosen B-life can be maintained or replaced (Figure 6). In this case of diagnostic data it concerns measurements on non-failed components. This raises the question which boundary values (B-lives) should be chosen when considering "safe" and "dangerous" limits. In next chapter with an experimental way B-lives will be improved based on HV components tests. 


\subsubsection{BOUNDARY EXPERIMENTAL ESTIMATION (BASED ON TESTS OF HV COMPONENTS)}

To verify the developed knowledge rules about boundary norm generation and aging processes several cable system components like termination with artificial defects were investigated in the laboratory. For aging investigation the following components were tested in the laboratory: terminations with an interface problem, e.g. internal discharges in stator and bushing insulation. Result will be shown for a cable termination which was tested in four degradation stages until breakdown. In particular, the partial discharges measured where related to a tracking mechanism. The slopes of the trend lines increase with aging. During 8 weeks of continuously applied voltage (in the range of $4 \mathrm{kV}$ up to $14 \mathrm{kV}$ ) the reading of PD amplitude was recorded. Figure 7 shows the measurement results.

With knowledge about the physical defects present in the component and the slope of the trend line condition marks were given. Combining the outcomes of the analyses mentioned in chapters 3 and tests the following procedure was applied to generate and to improve boundary values:

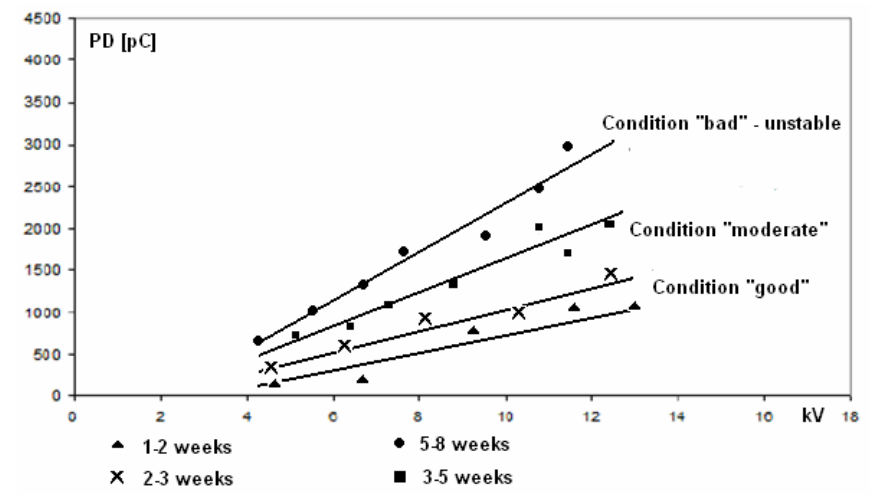

Figure 7. Experimental aging of cable termination [6,14].

The aging trend lines of the experimental data were indexed according to Table 1

1) The partial discharge magnitudes under consideration were put in a dataset,

2) The datasets were fitted to a statistical distribution,

3) The partial discharge magnitudes that lie in between the condition index regions were estimated,

4) The boundary values at which these estimated partial discharge magnitudes are situated were calculated and used in on-site data analysis.

The best fitting distribution for this dataset was the lognormal distribution as in Figure 8. The partial discharge magnitudes at nominal voltage used to calculate the boundaries values were estimated at $1200 \mathrm{pC}$ and $1700 \mathrm{pC}$. Using these values, the boundaries were situated at $40 \%$ and

Table 1. Condition index of the aging stages in the cable termination

\begin{tabular}{|r|r|l|}
\hline Week nr & PD average amplitude $[\mathrm{pC}]$ & condition \\
\hline 2 & 1000 & very good \\
\hline 4 & 1200 & good \\
\hline 6 & 1700 & moderate \\
\hline 8 & 3200 & bad \\
\hline
\end{tabular}

$80 \%$. Again these boundaries denoted the transition between the conditions as is visible.

It has been shown in $[6,14]$ that aging tests of other HV components (bushings and stators) resulted also in output data which fit reliability around $40 \%$ and $80 \%$.
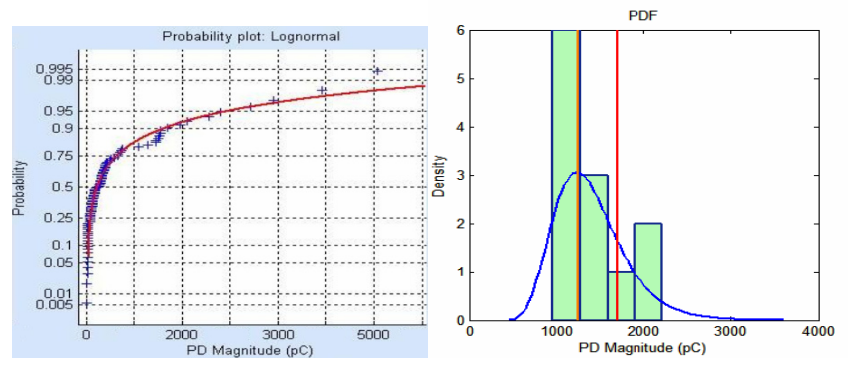

Figure 8. Fitting of the measurement data of the cable termination with boundaries at $40 \%$ and $80 \%$.

\section{APPLICATION OF STATISTICAL ANALYSIS TO POWER CABLES DIAGNOSTIC DATA (EXAMPLES)}

In this paragraph several examples of statistically calculated boundary values will be presented. The analysis performed on diagnostic data was used to obtain boundary values for the conditioning of $50 \mathrm{kV}$ oil-paper insulated power cables, $150 \mathrm{kV}$ external gas pressure $\mathrm{HV}$ power cables and $20 \mathrm{kV}$ distribution power cables both XLPE and PILC insulation. To see when the PD amplitude is reaching a critical limit and to generate knowledge rules boundary limits were taken and from the distributions the maximum allowable PD magnitude was obtained. This makes it possible to index the condition of the cable into good, moderate or bad and to give advice for future actions.

\subsection{KV MV DISTRIBUTION CABLE}

An existing database of measuring parameters consists of different types of $20 \mathrm{kV}$ distribution cables and accessories. All components were installed in a 47 years period of time with the oldest installed component was in 1958 and the newest in 2005. Because of this fact, analysis of population of certain type of joint, termination and insulation types gave valuable information of PD activity in installed serviced components and their aging status during service life. Therefore emphasis was put to three main groups of cables put under investigation:

First group consist of: 30 (3-phases) paper-mass insulated cables of different length,

Second group consist of: 9 (3-phase) XLPE insulated cables of different length,

Third group consist of 42 (3-phases) mixed insulation cables of different length.

In database available parameters like:

- $\mathrm{PD}$ magnitude at inception voltage: $\mathrm{PD}=\mathrm{PDIV}$,

- PD magnitude at service voltage: $\mathrm{PD}=\mathrm{Uo}$,

- $\mathrm{PD}$ magnitude at 1.7 value of service voltage: $\mathrm{PD}=1.7$ Uo

were used for the generation of boundary values of particular cable accessory type where PDs were detected and localized. 
Based on the assumption that MV cable systems data are represented by three separate insulation types and for each type several different accessory types can be specified with at least 9 samples showing PD activity, boundary representing norm values could be estimated (Figure 9). For this purpose input data representing PD parameters have been fitted with best fitting distribution of e.g. Normal, Lognormal, Weibull or Exponential and $40 \%$ and $80 \%$ boundaries have been estimated by statistical tools (Table 2).

In the proposal mentioned above the $40 \%$ and $80 \%$ boundaries estimation was based on experience obtained by laboratory aging experiments as described in section 3.4.1. Combining the outcomes of the analysis based on distributions fitting and laboratory aging test till breakdown of the component, it was possible to generate and tune boundary values and set $(40 \%$ and $80 \%$ ) values for these components types where PDs were found.
With this statistical approach it was possible to estimate the "dangerous" border values indicating ranges for three service conditions representing aging of the cable. Moreover, with known statistical boundaries obtained for $\mathrm{MV} / \mathrm{HV}$ components experimental condition indexes can be used to easily mark the typical condition situation during service life.

This condition assessment approach based on diagnostic parameters gives information about how big number of components had failure and how "statistically dangerous" are measured values e.g. for the termination type 1 (mass cables) "Status A" condition range regarding $\mathrm{PD}=\mathrm{PDIV}$ is specified between $364 \mathrm{pC}-40 \%$ and $1147 \mathrm{pC}-80 \%$ (Table 2). As a result, if investigated statistically component (termination type 1) reach $=$ PDIV maximum PD amplitude higher than 1147 pC automatically will be marked with index - condition bad. In this way respectively all cable system components (joints, terminations, insulation parts) can be judge.

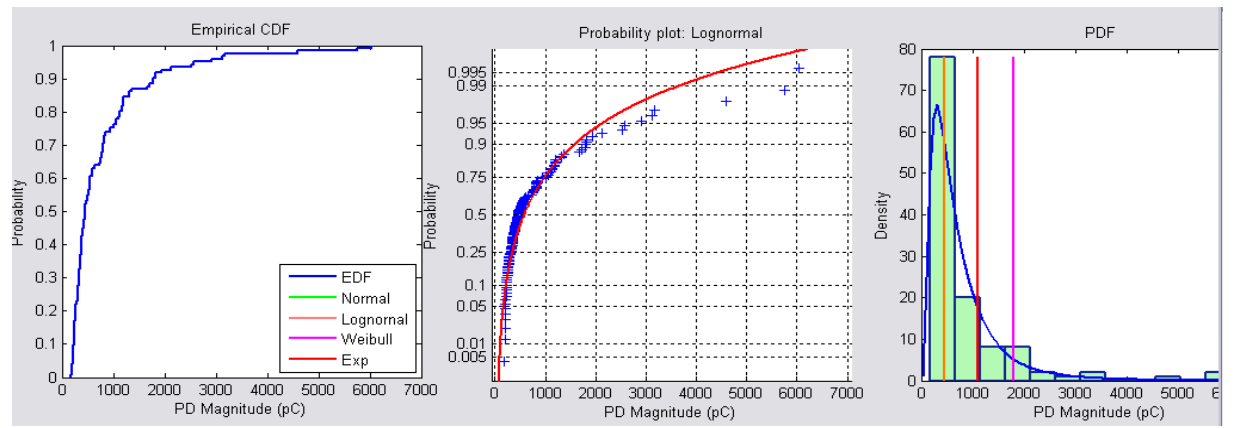

Figure 9. Example of boundary values calculation for PD- diagnostic parameter for $20 \mathrm{kV}$ termination type 1.

Table 2. Boundaries ( $40 \%$ and $80 \%$ ) values calculated for all components where PD were localized and number of component is 9 or higher.

( $\mathrm{x}$ - boundary estimation not possible or no samples).

\begin{tabular}{|c|c|c|c|c|c|c|c|c|c|}
\hline \multirow[b]{4}{*}{$\begin{array}{l}\text { Termination } \\
\text { Type } 1\end{array}$} & \multicolumn{9}{|c|}{ Mass insulated cabels $(20 \mathrm{kV})$} \\
\hline & \multicolumn{3}{|c|}{$\mathrm{Nr}$ of samples } & \multicolumn{2}{|c|}{ PD at PDV } & \multicolumn{2}{|c|}{ PD at Uo } & \multicolumn{2}{|c|}{ PD at $1,7 \mathrm{U}$} \\
\hline & $P D I V=<0,3 L$ & $0,3<P D I V<1,2 U_{0}$ & PDIV $=1,2 U_{0}$ & $40 \%$ & $80 \%$ & $40 \%$ & $80 \%$ & $40 \%$ & $80 \%$ \\
\hline & $x$ & 7 & & C & $147 \mathrm{pc}$ & & $x$ & 4420 & $630 \mathrm{c}$ \\
\hline Type 6 & $\hat{6}$ & 29 & 15 & $358 \mathrm{pC}$ & $9100 \mathrm{C}$ & $x$ & $\begin{array}{l}x \\
x\end{array}$ & $439 p c$ & $1062 \mathrm{pC}$ \\
\hline Joints & & & & & & & & & \\
\hline Type 1 & $x$ & 9 & 2 & $1394 \mathrm{pC}$ & $4263 p c$ & $\mathrm{x}$ & $\mathrm{x}$ & $1407 \mathrm{pc}$ & $4305 \mathrm{pC}$ \\
\hline Type 3 & $x$ & 10 & 4 & $389 \mathrm{pc}$ & $1055 \mathrm{pc}$ & $x$ & $x$ & $458 \mathrm{pC}$ & $1238 \mathrm{pc}$ \\
\hline Type 5 & $x$ & 60 & 47 & $464 \mathrm{pC}$ & $1122 \mathrm{pc}$ & $262 p c$ & $601 p c$ & $560 \mathrm{pC}$ & $1290 \mathrm{pC}$ \\
\hline Insulatic & & & & & & & & & \\
\hline $\begin{array}{l}\text { NA2XSFF } 2 Y \\
\text { NAEKBA }\end{array}$ & $x$ & $\begin{array}{l}12 \\
67\end{array}$ & $\begin{array}{c}4 \\
19\end{array}$ & $\begin{array}{l}457 \mathrm{pC} \\
389 \mathrm{pC}\end{array}$ & $\begin{array}{c}763 p C \\
978 p\end{array}$ & $\underset{341 p c}{x}$ & $\underset{715 p c}{x}$ & $\begin{array}{l}4820 \mathrm{C} \\
5430 \mathrm{C}\end{array}$ & $\begin{array}{l}1113 p \mathrm{c} \\
1363 \mathrm{p} C\end{array}$ \\
\hline
\end{tabular}

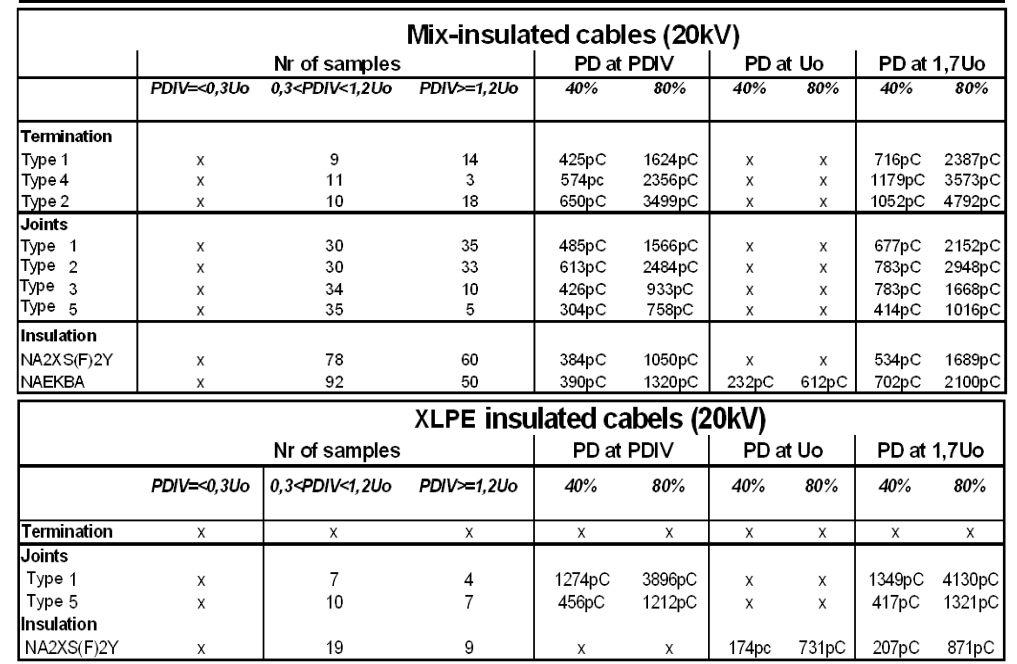


4.2 $50 \mathrm{KV}$ OIL-FILLED HV POWER TRANSMISSION CABLE

Input data representing oil-filled power cables consist of eleven ( 3 phase) $50 \mathrm{kV}$ oil-filled power cables circuits. All circuits were installed in Holland between 1950 and 1993. Discussion regarding the variation of two diagnostic parameters $\tan \delta$ and $\Delta \tan \delta$ has been done (Table 3).

Based on the fact that all single cable sections (phases) are representing the same type of insulation (with exception of circuit 3 the presence of XLPE and PILC mixed insulation is not significant) for dielectric losses measurements $\Delta \tan \delta$ and $\tan \delta$ statistical boundary have been estimated (including confidence bounds) as described in paragraph 3.4. Table 4 presents diagnostic parameters values such as estimated confidence bounds and $40 \%$ and $80 \%$ boundary values for tan $\delta$ and $\Delta \tan \delta$. From this, it follows that there is no overlap between calculated confidence bounds ranges at each confidence level (as it is visible with small data population of $150 \mathrm{kV}$ cables, described later).
This is a result of a larger amount of input data used for statistic estimation. With larger amount of input data boundary values for $\tan \delta$ values can be estimated with higher accuracy. Figure 10 presents example of

of boundary values calculation for Tan $\delta=$ Uo (A) and 1.7xUo (B).

Based on estimated boundary values for $\tan \delta$ and $\Delta \tan \delta$ and obtained circuit data (Table 3 ) it can be derived:

- that phase L1, L2 (circuit 1) and phase L2, L3 (circuit 8) are classified as most deviating (condition "bad" for $\Delta$ tan $\delta$ and condition "moderate" for $\tan \delta$ - condition aged),

- phase L1, L2 (circuit 2) and L1, L2, L3 (circuit 9) classified as most deviating (condition "bad"), Explanation of relatively high $\tan \delta$ (circuit 9) not possible yet, circuit 10 shows the lowest aging condition "good" for all phases.150 KV external gas pressure $\mathrm{HV}$ power transmission cable

Input data representing oil-filled power cables consist of three external gas pressure HV power cables (3-phases) 150

Table 3. Numeric values of diagnostic parameters.

\begin{tabular}{|c|c|c|c|c|c|c|c|c|c|c|c|c|c|c|c|}
\hline \multirow[b]{2}{*}{ Condition parameter } & \multicolumn{3}{|c|}{ Circuit 1} & \multicolumn{3}{|c|}{ Circuit 2} & \multicolumn{3}{|c|}{ Circuit 3} & \multicolumn{3}{|c|}{ Circuit 4} & \multicolumn{3}{|c|}{ Circuit 5} \\
\hline & L1 & L2 & L3 & L1 & L2 & L3 & L1 & L2 & L3 & L1 & L2 & L3 & L1 & L2 & L3 \\
\hline Tan ס @ Uo [\%] & 0.28 & 0.28 & 0.27 & 0.37 & 0.36 & 0.30 & 0.24 & 0.25 & 0.24 & 0.10 & 0.10 & 0.10 & 0.12 & 0.12 & 0.11 \\
\hline $\operatorname{Tan} \delta @ 1.7 \cup \circ[\%]$ & 0.38 & 0.37 & 0.34 & 0.42 & 0.42 & 0.37 & 0.30 & 0.30 & 0.27 & 0.16 & 0.18 & 0.16 & 0.20 & 0.20 & 0.19 \\
\hline$\Delta \operatorname{Tan} \delta[\%]$ & 0.10 & 0.094 & 0.071 & 0.054 & 0.055 & 0.075 & 0.064 & 0.049 & 0.039 & 0.06 & 0.075 & 0.06 & 0.076 & 0.075 & 0.076 \\
\hline PD @ Uo [pC] & 24 & 5 & 15 & 50 & 340 & 230 & 750 & 1000 & 880 & 11000 & 12000 & 12000 & 5000 & 5000 & 6000 \\
\hline PD @ 1.7Uo[pC] & 26 & 6 & 18 & 300 & 450 & 500 & $\mathbf{X}$ & 1800 & 1800 & 30000 & 35000 & 36000 & 24000 & 26000 & 30000 \\
\hline
\end{tabular}

\begin{tabular}{|c|c|c|c|c|c|c|c|c|c|c|c|c|}
\hline \multirow[b]{2}{*}{ Condition parameter } & \multicolumn{3}{|c|}{ Circuit 6} & \multicolumn{3}{|c|}{ Circuit 7} & \multicolumn{3}{|c|}{ Circuit 8} & \multicolumn{3}{|c|}{ Circuit 9} \\
\hline & L1 & L2 & L3 & L1 & L2 & L3 & L1 & L2 & L3 & L1 & L2 & L3 \\
\hline Tan ס @ Uo [\%] & 0.14 & 0.14 & 0.14 & 0.15 & 0.15 & 0.16 & 0.29 & 0.29 & 0.28 & 0.86 & 0.72 & 0.59 \\
\hline Tan ס @ 1.7Uo [\%] & 0.21 & 0.21 & 0.21 & 0.22 & 0.22 & 0.22 & 0.36 & 0.37 & 0.37 & 0.82 & 0.65 & 0.55 \\
\hline$\Delta \operatorname{Tan} \delta[\%]$ & 0.077 & 0.072 & 0.076 & 0.070 & 0.069 & 0.059 & 0.076 & 0.083 & 0.088 & 0.043 & 0.068 & 0.046 \\
\hline PD @ Uo [pC] & 20 & 34 & 24 & 9 & 8 & 4 & 30 & 30 & 35 & 20 & 40 & 42 \\
\hline PD @ 1.7Uo [pC] & 30 & 36 & 37 & 10 & 9 & 5 & 31 & 32 & 35 & 38 & 42 & 43 \\
\hline
\end{tabular}

\begin{tabular}{|c|c|c|c|c|c|c|}
\hline & \multicolumn{3}{|c|}{ Circuit 10} & \multicolumn{3}{|c|}{ Circuit 11} \\
\hline Condition parameter & L1 & L2 & L3 & L1 & L2 & L3 \\
\hline Tan ס @ Uo [\%] & 0.22 & 0.21 & 0.21 & 0.33 & 0.32 & 0.32 \\
\hline Tan $\delta @ 1.2 \cup o[\%]$ & 0.25 & 0.24 & 0.24 & 0.35 & 0.35 & 0.34 \\
\hline$\Delta \operatorname{Tan} \delta[\%]$ & 0.030 & 0.030 & 0.030 & 0.020 & 0.030 & 0.020 \\
\hline PD @ Uo [pC] & 25 & 30 & 30 & 3 & 5 & 4 \\
\hline PD @ $1.2 \cup \circ[p C]$ & 30 & 30 & 30 & 5 & 5 & 5 \\
\hline
\end{tabular}

A

Empinal COF

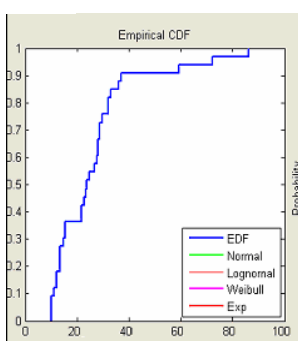

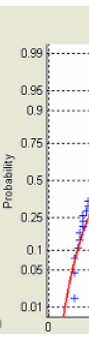

\section{B}
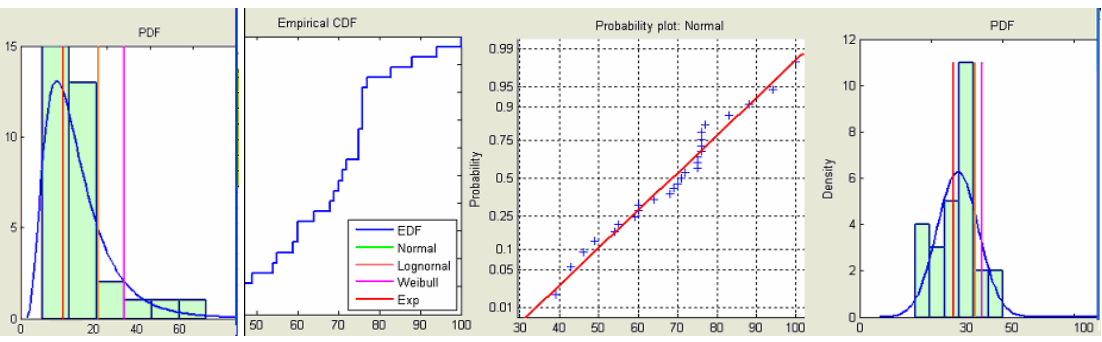

Figure 10. Example of boundary values calculation for $\operatorname{Tan} \delta=\mathrm{Uo}(\mathrm{A})$ and $1.7 \mathrm{Uo}$ (B).

Table 4. Numeric values of confidence bounds for $40 \%$ and $80 \%$ boundary $=50 \%, 75 \%, 90 \% 95 \% 99 \%$ confidence level.

\begin{tabular}{|c|c|c|c|c|c|c|c|c|c|c|c|}
\hline & & $\overline{99}$ & & 950 & & & D\% & & & & \\
\hline & Value & LB & UB & $\overline{\mathrm{LB}}$ & UB & LB & UB & LB & UB & LB & UB \\
\hline Boundary:40\% & $0.196 \%$ & $0.152 \%$ & $0.253 \%$ & $0.161 \%$ & \begin{tabular}{|l|}
$0.238 \%$ \\
\end{tabular} & $0.166 \%$ & \begin{tabular}{|l|}
$0.230 \%$ \\
\end{tabular} & $0.175 \%$ & $0.219 \%$ & $0.183 \%$ & 0.205 \\
\hline Boundary: $80 \%$ & $0.361 \%$ & $0.269 \%$ & $0.485 \%$ & $0.289 \%$ & \begin{tabular}{|l|}
$0.452 \%$ \\
\end{tabular} & $\begin{array}{l}0.299 \% \\
\end{array}$ & $0.436 \%$ & $0.317 \%$ & $0.412 \%$ & $0.335 \%$ & 0.390 \\
\hline
\end{tabular}

\begin{tabular}{|c|c|c|c|c|c|c|c|c|c|c|c|}
\hline & & & $9 \%$ & & & & & & & & \\
\hline & Value & LB & JUB & LB & TUB & LB & UB & LB & UB & & UB \\
\hline \begin{tabular}{|l|} 
Boundary:40\% \\
\end{tabular} & $0.263 \%$ & \begin{tabular}{|l}
$0.219 \%$ \\
\end{tabular} & $0.316 \%$ & $0.229 \%$ & \begin{tabular}{|l|} 
\\
\end{tabular} & $0.234 \%$ & \begin{tabular}{|l|}
$0.296 \%$ \\
\end{tabular} & \begin{tabular}{|l|}
$0.243 \%$ \\
\end{tabular} & $0.286 \%$ & $0.251 \%$ & 0.276 \\
\hline \begin{tabular}{|l|} 
Boundary: $80 \%$ \\
\end{tabular} & $0.408 \%$ & $0.330 \%$ & $0.503 \%$ & $0.347 \%$ & $0.479 \%$ & $0.356 \%$ & \begin{tabular}{|l|}
$0.466 \%$ \\
\end{tabular} & $0.371 \%$ & $0.448 \%$ & $0.386 \%$ & 0.431 \\
\hline
\end{tabular}

\begin{tabular}{|c|c|c|c|c|c|c|c|c|c|c|c|}
\hline \multicolumn{12}{|c|}{$\Delta \tan \delta$} \\
\hline & & & $9 \%$ & & $5 \%$ & & $0 \%$ & & $5 \%$ & & $\%$ \\
\hline & Value & LB & JUB & LB & JUB & LB & UB & LB & UB & LB & UB \\
\hline \begin{tabular}{|l|} 
Boundary:40\% \\
\end{tabular} & $0.056 \%$ & \begin{tabular}{|l|l|}
$0.045 \%$ \\
\end{tabular} & $0.667 \%$ & $0.048 \%$ & \begin{tabular}{|l|}
$0.064 \%$ \\
\end{tabular} & $0.049 \%$ & $0.063 \%$ & $0.051 \%$ & $0.060 \%$ & $0.053 \%$ & $0.059 \%$ \\
\hline \begin{tabular}{|l|} 
Boundary: $80 \%$ \\
\end{tabular} & $0.080 \%$ & \begin{tabular}{|l|}
$0.068 \%$ \\
\end{tabular} & $0.092 \%$ & $0.071 \%$ & $0.089 \%$ & $0.072 \%$ & $0.088 \%$ & $0.075 \%$ & $0.085 \%$ & $0.077 \%$ & 0.083 \\
\hline
\end{tabular}


$\mathrm{kV}$ and one (3-pahses) section of $110 \mathrm{kV}$. All circuits have been installed between 1950 and 1990. No maintenance history was available for the particular circuit.

PD measurements were performed at 0.4 Uo up to Uo PD, numeric values are presented in Table 5 .

Next measuring result regards to dielectric losses diagnostics and indicates overall insulation condition. By increasing the test voltage and comparing losses of measured phases it was observed that measured $\tan \delta$ was in range of $<0.30 \%$ $0.49 \%>\left(30 \times 10^{-4}-49 \times 10^{-4}\right)$. The highest values were observed in phase L1 (circuit 1) at every increment of testing voltage.

By comparing values of $\tan \delta$ at consecutive test voltage levels $\Delta \tan \delta$ in function of test voltage, has been derived. It follows that phase L1 represents the highest values of this parameter (Table 5). For this purpose both statistical distributions have been fitted and $40 \%$ and $80 \%$ boundaries have been calculated (Figure 11) including the confidence bounds at $50 \%, 75 \%, 90 \%, 95 \%$ and $99 \%$ confidence level (Table 6).

This confidence interval represents the maximum error expected at this calculated boundaries norm. The lower the confidence level the lower maximum error is. From the Table 6 follows that there is an overlap between $40 \%$ boundary and the $80 \%$ boundary for $\tan \delta=0.4 \mathrm{Uo}$ and Uo as well as for $\Delta \tan \delta$. The overlap between boundary $40 \%$ and $80 \%$ is present only at $99 \%$ and $95 \%$ confidence level. This overlap is due to small number of data as used for $110 \mathrm{kV}$ and $150 \mathrm{kV}$ gas pressurized cables.

It follows from this evaluation that based on both parameters $\tan \delta$ and $\Delta \tan \delta$ :

- phase L1 (section 1) is classified as most deviating (condition moderate for $\Delta \tan \delta$ and condition "bad" for $\tan \delta$, condition aged),

- phase L1, L2, L2 (section 3) are deviating (condition "moderate"),

- all phases (section 2) show no deviation (condition "good' condition no aging),

- two phases (section 4) show no deviation ( condition "good" no aging), phase L1 (index 6, condition "moderate").

\section{CONCLUSIONS}

Based on the research results as discussed in this contribution the following can be concluded:

1. Service aged power cables are important assets and there is an increasing need to obtain information about actual condition by applying integral and defect related on-site diagnostics.

2. Partial discharges, dielectric losses diagnosis can be used for non-destructive on-site testing and diagnosis of new and service aged power cables

Table 5. Numeric values of diagnostic parameters.

\begin{tabular}{|c|c|c|c|c|c|c|c|c|c|c|c|c|}
\hline \multirow[b]{2}{*}{ Diagnostic parameter } & \multicolumn{3}{|c|}{ Section 1} & \multicolumn{3}{|c|}{ Section 2} & \multicolumn{3}{|c|}{ Section 3} & \multicolumn{3}{|c|}{ Section 4} \\
\hline & L1 & L2 & L3 & L1 & L2 & L3 & L1 & L2 & L3 & L1 & L2 & L3 \\
\hline Tan ס @ 0.4Uo [\%] & 0.37 & 0.32 & 0.30 & 0.39 & 0.37 & 0.35 & 0.35 & 0.35 & 0.36 & 0.37 & 0.37 & 0.34 \\
\hline Tan ס @ Uo [\%] & 0.50 & 0.41 & 0.42 & 0.44 & 0.45 & 0.43 & 0.48 & 0.47 & 0.47 & 0.47 & 0.46 & 0.45 \\
\hline PD level @ 0.4Uo [pC] & 13 & 2 & 3 & 112 & 145 & 138 & 40 & 13 & 28 & 21 & 18 & 42 \\
\hline PD level @U & 51 & 74 & 44 & 113 & 166 & 250 & 160 & 106 & 72 & 106 & 86 & 101 \\
\hline$\Delta \operatorname{Tan} \delta[\%]$ & 0.125 & 0.09 & 0.12 & 0.051 & 0.074 & 0.089 & 0.132 & 0.121 & 0.108 & 0.105 & 0.098 & 0.103 \\
\hline
\end{tabular}

A

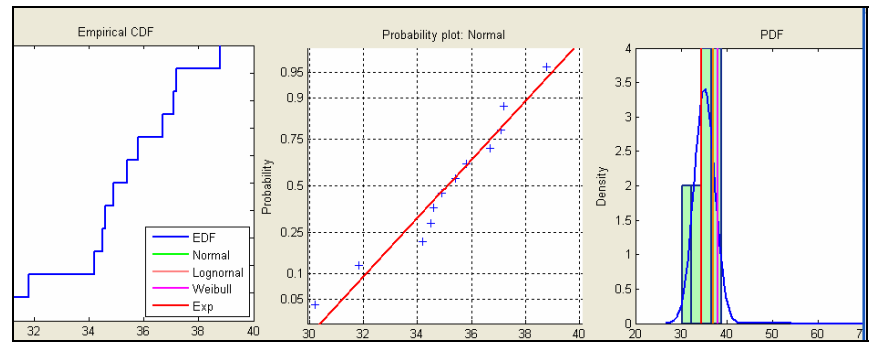

B

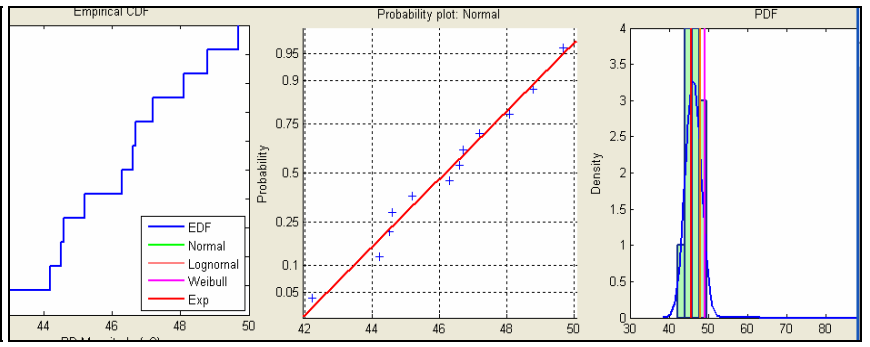

Figure 11. Example of boundary values calculation for Tan $\delta=0.4$ Uo (A) and Uo (B).

Table 6. Numeric values of confidence bounds for $40 \%$ and $80 \%$ boundary $=50 \%, 75 \%, 90 \% 95 \% 99 \%$ confidence level.

\begin{tabular}{|c|c|c|c|c|c|c|c|c|c|c|c|c|}
\hline & & \multicolumn{3}{|c|}{$99 \%$} & \multicolumn{2}{|c|}{$95 \%$} & \multicolumn{2}{|c|}{$90 \%$} & \multicolumn{2}{|c|}{$75 \%$} & \multicolumn{2}{|c|}{$50 \%$} \\
\hline & Value & LB & & UB & $\mathrm{LB}$ & UB & $\angle B$ & UB & $\angle B$ & UB & LB & UB \\
\hline Boundary:40\% & $0.345 \%$ & & $326 \%$ & $0.364 \%$ & $0.330 \%$ & $0.359 \%$ & $0.333 \%$ & $0.357 \%$ & $0.336 \%$ & $0.353 \%$ & $0.340 \%$ & 0.350 \\
\hline Boundary: $80 \%$ & $0.372 \%$ & & $0.350 \%$ & $0.394 \%$ & $0.355 \%$ & $0.389 \%$ & $0.358 \%$ & $0.386 \%$ & $0.362 \%$ & $0.382 \%$ & $0.366 \%$ & 0.37 \\
\hline \multicolumn{13}{|c|}{$\tan \delta @$ Uo } \\
\hline & & \multirow{2}{*}{\multicolumn{3}{|c|}{$99 \%$}} & \multicolumn{2}{|c|}{$95 \%$} & \multicolumn{2}{|c|}{$90 \%$} & \multicolumn{2}{|c|}{$75 \%$} & \multicolumn{2}{|c|}{$50 \%$} \\
\hline & Value & & & UB & LB & UB & $\mathrm{LB}$ & UB & LB & UB & LB & UB \\
\hline Boundar & $0.456 \%$ & & $439 \%$ & $0.473 \%$ & $0.443 \%$ & $0.469 \%$ & $0.445 \%$ & $0.467 \%$ & $0.448 \%$ & $4 \%$ & $0.451 \%$ & 0.46 \\
\hline Boundary: $80 \%$ & $0.481 \%$ & & $0.460 \%$ & $0.502 \%$ & $0.465 \%$ & $0.497 \%$ & $0.468 \%$ & $0.494 \%$ & $0.472 \%$ & $0.490 \%$ & $0.476 \%$ & 0.486 \\
\hline
\end{tabular}

$\Delta \tan \delta$

\begin{tabular}{|c|c|c|c|c|c|c|c|c|c|c|c|}
\hline & & $99 \%$ & & & $5 \%$ & $\overline{90}$ & & & & $\overline{50^{\circ}}$ & \\
\hline & Value & LB & UB & $\angle B$ & JUB & $\angle B$ & $\overline{\mathrm{JB}}$ & LB & $\overline{U B}$ & $\overline{L B}$ & $\mathrm{UB}$ \\
\hline 800 & $0.104 \%$ & $\%$ & 0.12 & $\%$ & 0.1 & $0.089 \%$ & 0.1 & $0.094 \%$ & 0.1 & $0.098 \%$ & \\
\hline unda & $0.135 \%$ & $0.109 \%$ & $0.160 \%$ & $0.115 \%$ & $0.154 \%$ & $0.118 \%$ & 0.15 & $0.123 \%$ & $0.146 \%$ & $0.128 \%$ & 0.141 \\
\hline
\end{tabular}


3. Statistical analysis can be a useful tool for analysing different types of diagnostic data and life time data.

4. Estimation of boundary values for particular cable system components can result in reference values, so called borders between "good", "moderate" and "bad" condition status.

5. Tracing the boundary values ranges it is possible to reduce un-expected cable failure by taking decisions: replace, maintain, refurbish or measure (check) again.

6. With statistical approach to diagnostic data and boundary estimation on-site measuring criteria can be setup and reference developed.

7. Knowledge rules about aging processes and physical PD background knowledge is necessary to estimate B-lives values.

8. To support AM decision processes, based on field experiences and by using sufficient amount of diagnostic data for different types of insulation and accessories statistical analysis can be used.

\section{REFERENCES}

[1] J. Popma and J. Pellis, "Diagnostics for high voltage cable systems", proceedings ERA Conf. HV Plant Life Extension, pp. 84-96 [Note(s) : env. 350 p.] (6 ref.) ISBN 0-7008-0723-323-24 November, Belgium, 2000.

[2] CIGRE WG 21.05, "Diagnostic Methods for HV Paper Cables and Accessories", Electra No. 176, 1998.

[3] F.J. Wester, "Condition Assessment of Power Cables using Partial discharge Diagnosis at Damped AC Voltages", ISBN 90-8559019-1, Ph.D. theses TU Delft, The Netherlands, 2004.

[4] E. Gulski, F.J. Wester, J.J. Smit and E. Groot, "Insulation Condition Assessment by Non-destructive Diagnosis of Distribution Power Cables", XIV ${ }^{\text {th }}$ Intern. Symposium on High Voltage Engineering, Tshinghua University, paper G007, Beijng, China, 2005.

[5] R.A Jongen, E. Gulski and J.J Smit, "Statistical Analysis of Diagnostic - and Life Time Data of High Voltage Components", $8^{\text {th }}$ Intern. Power Engineering Conf, IPEC, pp. 455-459, Singapore, 2007.

[6] B. Quak, Information Strategy for Decision Support in Maintaining High Voltage Infrastructures, ISBN 978-90-8559334-8, 2007, Ph.D. thesis TU Delft, The Netherlands 2008.

[7] E. Gulski, J.J. Smit, P. Cichecki, P.P. Seitz, B. Quak, F. de Vries and F. Petzold, "Insulation Diagnosis of HV Power Cables", Jicable, $7^{\text {th }}$ Intern. Conf. Insulated Power Cables, Versailles, France, Paper 51, 2007.

[8] F.H. Kreuger, Industrial High Voltage, Coordinating, Testing, Measuring, Delft University Press, The Netherlands, pp. 114-115, 1992.

[9] P. Osvath and Thus M. Zahn, "Polariation spectrum analysis for diagnosis of oil/paper insulation systems", IEEE Intern. Sympos. Electr. Insul. (ISEI), Pittsburgh, PA, USA, 1994.

[10] R.A. Abernethy, The New Weibull Handbook. 4th ed. North Palm Beach, Florida, USA, 2003.

[11] R. Billinton and R.N. Allan, Reliability Evaluation in Engineering Systems. Concepts and Techniques, London, UK, Plenum Press, 1992.
[12] P.D.T. O'Connor, Practical Reliability Engineering, 4th ed., Chichester, J. Wiley, 2003

[13] D. Kececioglu, Reliability and Life Testing Handbook, Vol. I, Englewood Cliffs, New Jersey, USA, 1993.

[14] E. Gulski, J.J. Smit, B. Quak, O. Piepers, F.J. Wester and F. de Vries, "Advanced partial discharges data analysis of distribution power cables", Intern. Conf. Condition Monitoring and Diagnosis (CMD), Changwon, pp. 1-6, Korea, 2006.

[15] E.Gulski, R.A. Jongen and J. Maksymiuk, "Analysis of Condition Data to Support Asset Management Decision Processes", Przeglad Elektrotechniczny, Vol. R83, pp. 133-142, 2007.

[16] R.A. Jongen, P.H.F. Morshuis, E. Gulski, J.J. Smit, J. Maksymiuk and A.L.J. Janssen, "Application of statistical methods for making maintenance decisions within power utilities", IEEE Electr. Insul. Mag., Vol. 22, No. 6, pp.24-35, 2006.

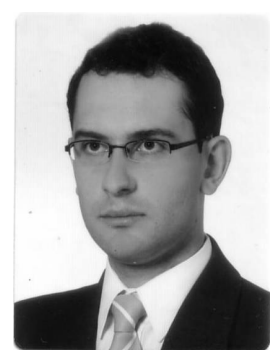

Piotr Cichecki was born in 1982 in Poland. He received the M.Sc. degree in electrical engineering in 2006 from Warsaw University of Technology. In the same year he joined High Voltage Technology \& Management Institute at the Delft University of Technology where he is pursuing the Ph.D. degree. His main field of research is high voltage power cables diagnostics with different on-site methods.

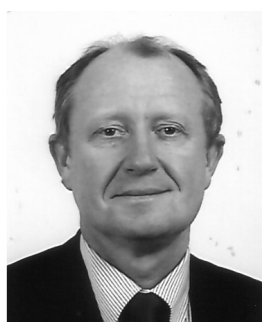

Edward Gulski received the M.Sc. degree in information technology in 1982 from Dresden University of Technology in Germany. In 1991 he received the Ph.D. degree from Delft University of Technology in the Netherlands. In 2004 he received the Doctor Habilitatus degree from Warsaw University of Technology in Poland. At present he is working at Delft University of Technology, and as a professor involved in education and research in the field of insulation diagnosis of HV components and Asset Management. He is a member of the Executive Board of KSANDR organization and responsible for research in education. He is chairmen of Cigre WG D1.17 "HV asset condition assessment tools, data quality, and expert systems", member of Cigre Study Committee D1 "Materials and Emerging Test Technologies", convener of Cigre TF D1.33.03 "Partial Discharges Measurements", member of the IEEE Insulating Conductors Committee, member of IEEE WG 400.3, chairmen of the IEEE Discussion Group F05. He is author and co-author of more than 300 publications and three books in the field of HV diagnosis and Asset Management.

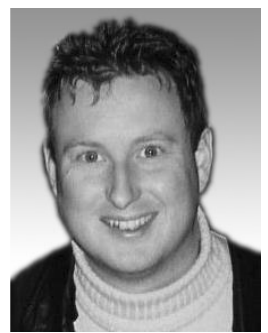

Rogier A. Jongen was born in 1976 in Nijmegen, The Netherlands. He received the M.Sc. degree in electrical engineering from the Delft University of Technology in 2004. His thesis project, 'PD diagnostics for transformers, ultra high frequency measurements as diagnostic tool', discusses the application of an ultra high frequency measuring system for the detection, localisation and identification of partial discharges in a transformer. Since 2005, he is doing Ph.D. degree research in the group of High Voltage Technology and Management of the Delft University of Technology. He is doing his research on the remaining life estimation of HV infrastructures, based on failure analysis. This can be used for AM decision support on overhaul or replacement of HV equipment. 


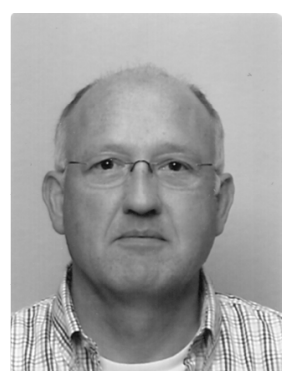

Johan J. Smit graduated in 1974 in experimental physics at the University of Amsterdam and in 1979 he received the Ph.D. degree at the State University of Leiden. $\mathrm{He}$ was employed in different research/management functions during two decades at KEMA's testing, consultancy and engineering company, Arnhem NL. Furthermore he was supervisory board member of the power grid company of South Holland, Director of Education in Electrical Engineering and general chair of the International Symposium on High Voltage Engineering in 2003. From 1996 as a fulltime professor he is director of High Voltage Technology \& Management Institute at the Delft University of Technology. He is chairman of the executive board of the KSANDR, organization for Knowledge Sharing AND Research in the field of energy infrastructures. He is the Dutch representative in IEC TC112 (Electrical Insulation Systems), former chairman of SC D1 (Materials and Emerging Technologies), active in D1 and B3 advisory boards, and convener of WG B3.06 (substation management). In September 2004 he has been awarded with the honorary membership of CIGRE, Paris.

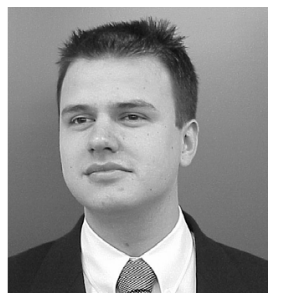

Ben Quak received the M.Sc. degree in electrical engineering from the Delft University of Technology in 2001. His thesis project 'Database support for condition based maintenance on distribution power cable' handled generation and application of knowledge rules on collections of measurement data. From 2001 till 2005 he was a researcher in the HV Technology and Management group of Delft University of Technology working towards his $\mathrm{PhD}$ thesis. In co-operation with Dutch TSO TenneT his activities concentrated on research for tools and systems for asset management of high voltage infrastructures. He is active in Cigré as the secretary of WG D1.17 (Asset condition assessment tools, data quality, and expert systems.) Currently he is CTO of Seitz Instruments AG, Switzerland.

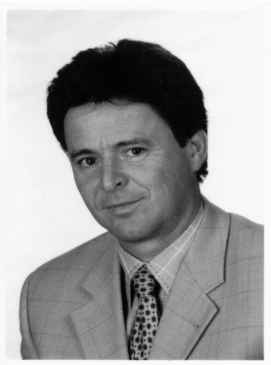

Frank Petzold was born in 1955 in Dresden (Germany). From 1975 to 1979 he studied electrical engineering at the High Voltage Institute of the Technical University Dresden. In 1984 he received the Ph.D. degree from High Voltage Institute of Technical University Dresden. The title of his thesis was "Influence of several stabilizers on tree-inception in XLPE insulations". From 1984 to 1990 he was chief of the development department in the cable factory KWO Schwerin (Germany). From 1990 to 1999 he worked in cooperation with SEBA Dynatronic with his own consulting company for cable fault location and leak detection in Germany. Since 2000 he is Vice President of SEBA Dynatronic GmbH Germany, responsible for research and development and technical marketing of the SEBAKMT group. Mr. Petzold is an active Member of several working groups in IEC; CIGRE and IEEE.

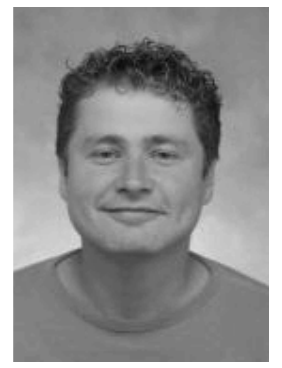

Frank de Vries graduated from the Alkmaar Polytechnic School in 1997 and started working at the utility Nuon as a high voltage engineer. Special fields of interest included condition assessments on MV and HV components. In 2000, together with the Technical University of Delft, he was involved in the implementation of OWTS technique on cables at Nuon and other grid owners. He also works as a component engineer at KSANDR, an independent organization for knowledge sharing and research. 